\title{
CONTRACTION GROUPS FOR TIDY AUTOMORPHISMS OF TOTALLY DISCONNECTED GROUPS
}

\author{
HELGE GLÖCKNER \\ TU Darmstadt, Fachbereich Mathematik AG 5, Schlossgartenstr. 7, 64289 Darmstadt, Germany \\ e-mail: gloeckner@mathematik.tu-darmstadt.de
}

(Received 3 September, 2004; accepted 18 March, 2005)

\begin{abstract}
In this note, we show that results of U. Baumgartner and G. A. Willis concerning contraction groups of automorphisms of metrizable totally disconnected, locally compact groups remain valid also in the non-metrizable case, if one restricts attention to automorphisms for which small tidy subgroups exist.
\end{abstract}

2000 Mathematics Subject Classification. Primary 22D05. Secondary 22D40, $22 \mathrm{D} 45$.

Given an automorphism $\alpha$ of a locally compact group $G$, its contraction group $U_{\alpha}$ is the group of all $x \in G$ such that $\lim _{n \rightarrow \infty} \alpha^{n}(x)=1$. Contraction groups arise in the study of semistable convolution semigroups on second countable groups [6], [8]. Contraction groups in $p$-adic Lie groups have been investigated in [2], and recently general results for metrizable totally disconnected groups $G$ were obtained, using the concept of a tidy subgroup (see [10], [11]) as a tool [1]. We recall the definition.

DefinItion 1 . Let $G$ be a totally disconnected, locally compact group and $\alpha$ a (bicontinuous) automorphism of $G$. A compact, open subgroup $V$ of $G$ is called tidy for $\alpha$ if

T1 $V=V_{+} V_{-}$, where $V_{ \pm}:=\bigcap_{n \in \mathbb{N}_{0}} \alpha^{ \pm n}(V)$; and

T2 The subgroups $V_{++}:=\bigcup_{n \in \mathbb{N}_{0}} \alpha^{n}\left(V_{+}\right)$and $V_{--}:=\bigcup_{n \in \mathbb{N}_{0}} \alpha^{-n}\left(V_{-}\right)$are closed in $G$.

In particular, twelve equivalent characterizations of closedness of $U_{\alpha}$ were given in [1, Theorem 3.32], for metrizable $G$. Notably, $U_{\alpha}$ is closed if and only if $G$ has small subgroups tidy for $\alpha$. The latter property is crucial for our discussion of the non-metrizable case. It is convenient to use the following terminology.

DEFINITION 2. An automorphism $\alpha$ of a totally disconnected, locally compact group $G$ is called tidy if $G$ has small subgroups tidy for $\alpha$.

While every automorphism of a $p$-adic Lie group is tidy [1, Remark 3.33(2)], already for automorphisms of Lie groups over local fields of positive characteristic tidiness is an important additional regularity property, which is not always satisfied [4]. In this setting, the tidiness property characterizes those analytic automorphisms whose scale can be calculated on the Lie algebra level [4]. Presuming tidiness of the automorphisms

\footnotetext{
${ }^{1}$ The present research was supported by DFG grant 447 AUS-113/22/0-1 and ARC grant LX 0349209. The author thanks U. Baumgartner and G. A. Willis for discussions concerning the tidying procedure.
} 
involved, it is frequently possible to extend results for $p$-adic Lie groups (as in [3], [5] or [9]) to the case of positive characteristic [4].

In this note, we describe consequences of the tidiness property. Based on techniques from [1], we establish two lemmas which allow us to transfer many of the results from [1] to non-metrizable groups, in the special case of tidy automorphisms. We also show that an automorphism $\alpha$ is tidy if and only if its restriction $\left.\alpha\right|_{M_{\alpha}}$ to the "Levi factor" $M_{\alpha}:=\left\{x \in G: \alpha^{\mathbb{Z}}(x)\right.$ is relatively compact $\}$ is tidy.

Throughout the article, $G$ is a totally disconnected, locally compact topological group and $\alpha \in \operatorname{Aut}(G)$.

\section{Definition 3. (See [1].)}

(a) The contraction group is $U_{\alpha}:=\left\{x \in G: \alpha^{n}(x) \rightarrow 1\right.$ as $\left.n \rightarrow \infty\right\}$.

(b) $U_{0}:=\overline{U_{\alpha}} \cap \overline{U_{\alpha^{-1}}}$, using the closures of $U_{\alpha}$ and $U_{\alpha^{-1}}$.

(c) The parabolic subgroup is $P_{\alpha}:=\left\{x \in G: \alpha^{\mathbb{N}}(x)\right.$ is relatively compact $\}$.

(d) The Levi factor is $M_{\alpha}:=P_{\alpha} \cap P_{\alpha^{-1}}$.

(e) If $V \subseteq G$ is a subgroup tidy for $\alpha$, we set $V_{0}:=V_{+} \cap V_{-}$, where $V_{+}$and $V_{-}$are as in Definition 1.

(f) The scale of $\alpha$ is defined as the index $s_{G}(\alpha):=\left[\alpha\left(V_{+}\right): V_{+}\right]$, where $V \subseteq G$ is a subgroup tidy for $\alpha$. It is known that tidy subgroups always exist, and that $s_{G}(\alpha)$ is well defined, independent of the choice of tidy subgroup (see [10]). The scale $s_{G}(\alpha)$ is a positive integer.

(g) Given a subgroup $H \subseteq G$ and $x \in G$, we write $\lim _{n \rightarrow \infty} \alpha^{n}(x)=1 \bmod H$ if, for every identity neighbourhood $V \subseteq G$, there exists $N \in \mathbb{N}$ such that $\alpha^{n}(x) \in V H$ for all $n \geq N .^{2}$ We write

$$
U_{\alpha / H}:=\left\{x \in G: \lim _{n \rightarrow \infty} \alpha^{n}(x)=1 \bmod H\right\} .
$$

Of course, $U_{0}$ (as in (b)) and $V_{0}$ (as in (e)) must not be confused. We now compile various facts which are essential for the rest of the paper. First, we recall from [1, Proposition 3.4] that $U_{\alpha}$ is a normal subgroup of $P_{\alpha}$. Next, let $V \subseteq G$ be a compact, open subgroup that is tidy for $\alpha$. Then $\alpha\left(V_{+}\right) \supseteq V_{+}$and $\alpha\left(V_{-}\right) \subseteq V_{-}$, whence $V_{-} \subseteq P_{\alpha}$ in particular. If $V$ is tidy, then $P_{\alpha} \cap V=V_{-}$by [1, Remark 3.1], whence $P_{\alpha}$ is closed. As a consequence, also $M_{\alpha}=P_{\alpha} \cap P_{\alpha^{-1}}$ is closed, and $V_{0}=V \cap M_{\alpha}$ if $V$ is tidy [10, Lemma 9]. The closedness of $M_{\alpha}$ is essential for the following; it ensures that also $M_{\alpha}$ is a totally disconnected, locally compact group. If $H \leq G$ is an $\alpha$-stable closed subgroup (i.e., $\alpha(H)=H$ ) and $V \subseteq G$ is tidy for $\alpha$, then there exists a subgroup $W \subseteq G$ tidy for $\alpha$ such that $W \subseteq V$ and $W \cap H$ is tidy for $\left.\alpha\right|_{H}\left[\mathbf{1 1}\right.$, Lemma 4.1]. Hence $\left.\alpha\right|_{H}$ is tidy if $\alpha$ is.

We now adapt [1, Theorem 3.8] to the non-metrizable case, for automorphisms such that $\left.\alpha\right|_{M_{\alpha}}$ is tidy (cf. also [7] and [2, Theorem 3.1], where analogous conclusions were achieved for real, respectively, $p$-adic Lie groups and compact $H$ ).

LEMMA 1. Let $H$ be an $\alpha$-stable closed subgroup of $G$. If $\left.\alpha\right|_{M_{\alpha}}$ is tidy, then

$$
U_{\alpha / H}=U_{\alpha} H
$$

\footnotetext{
${ }^{2}$ The definition in [1] only requires $\alpha^{n}(x) \in V H V$, but it is the above property which is really used in the proof of loc. cit. Theorem 3.8 (see p. 227, line 7 from below).
} 
Proof. The inclusion $U_{\alpha} H \subseteq U_{\alpha / H}$ is trivial. For the reverse inclusion, let $x \in U_{\alpha / H}$; we have to find $h \in H$ such that $x h \in U_{\alpha}$. Let $O$ be a compact, open subgroup of $G$ tidy for $\alpha$. By [1, Lemma 3.10], there exists $h_{0} \in H$ such that $x h_{0} \in U_{\alpha / H \cap O_{0}}$. Since $O_{0}=M_{\alpha} \cap O$, after replacing $H$ with $H \cap O_{0}$ and $x$ with $x h_{0}$ we may assume now without loss of generality that $H \subseteq M_{\alpha}$ and $H$ is compact. Let $\mathcal{B}$ be the set of compact open subgroups of $G$. For each $V \in \mathcal{B}$, [1, Lemma 3.10] yields $h_{V} \in H$ and $N_{V} \in \mathbb{N}$ such that $\alpha^{n}\left(x h_{V}\right) \in V$, for all $n \geq N_{V}$. Since $H$ is compact, the net $\left(h_{V}\right)_{V \in \mathcal{B}}$ has a cluster point $h \in H$. We claim that $\alpha^{n}(x h) \rightarrow 1$ as $n \rightarrow \infty$; i.e., $x h \in U_{\alpha}$. Given an identity neighbourhood $Y \subseteq G$, there exists an identity neighbourhood $W \subseteq G$ such that $W W \subseteq Y$; since $M_{\alpha}$ has small subgroups tidy for $\left.\alpha\right|_{M_{\alpha}}$, after shrinking $W$ we may assume that $W \cap M_{\alpha}$ is a compact open subgroup of $M_{\alpha}$ tidy for $\left.\alpha\right|_{M_{\alpha}}$. Then $H \cap W=H \cap M_{\alpha} \cap W=H \cap W_{0}$ is $\alpha$-stable. By the definition of $h$ as a cluster point, there exists $V \in \mathcal{B}$ with $V \subseteq W$ such that $h_{V}^{-1} h \in W$ and thus $h_{V}^{-1} h \in H \cap W$. For all $n \geq N_{V}$, we now obtain

$$
\alpha^{n}(x h)=\alpha^{n}\left(x h_{V}\right) \alpha^{n}\left(h_{V}^{-1} h\right) \in V \alpha^{n}(H \cap W)=V(H \cap W) \subseteq W W \subseteq Y .
$$

Hence indeed $\alpha^{n}(x h) \rightarrow 1$.

As a consequence of Lemma 1 just established, Proposition 3.7-Corollary 3.30 from [1] remain valid also for non-metrizable $G$, if one assumes in addition that $\left.\alpha\right|_{M_{\alpha}}$ is tidy. In fact, inspection shows that metrizability is never used directly in the proofs of these results, but only the validity of Equation (1) from above. The proof of Lemma 3.31 in [1], however, requires metrizability of $G$ for a second reason. The lemma exploits an algorithm for the construction of tidy subgroups described in [12, § 2]. This "tidying procedure" involves a certain subgroup $K$, whose definition we presently recall. If $G$ is metrizable, then $K=\overline{U_{\alpha} \cap P_{\alpha^{-1}}}$ (see [12, p. 4]), and this equality is used essentially in [1]. Unfortunately, it is not known whether equality persists for non-metrizable $G$. Therefore, to transfer [1, Lemma 3.31 and Theorem 3.32] to our setting, we first need to discuss $K$ in detail, assuming that $\left.\alpha\right|_{M_{\alpha}}$ is tidy. Let us recall the definition from $[12$, p. 4].

Definition 4. Write $\mathcal{K}_{O}:=\left\{x \in M_{\alpha}: \alpha^{n}(x) \in O\right.$ for all sufficiently large $\left.n\right\}$ and $K_{O}:=\overline{\mathcal{K}_{O}}$, for each compact open subgroup $O \subseteq G$. We define

$$
K:=\bigcap\left\{K_{O}: O \text { a compact open subgroup of } \mathrm{G}\right\} \text {. }
$$

Note that $\mathcal{K}_{O}=O_{--} \cap M_{\alpha}=\left(O \cap M_{\alpha}\right)_{--}$here.

LEMMA 2. If $\left.\alpha\right|_{M_{\alpha}}$ is tidy, then $K=U_{0}=\{1\}$.

Proof. Let us show first that $K \subseteq \bigcap\left\{W_{--}: W \in \mathcal{T}\left(\left.\alpha\right|_{M_{\alpha}}\right)\right\}$, where $\mathcal{T}\left(\left.\alpha\right|_{M_{\alpha}}\right)$ is the set of subgroups $W \subseteq M_{\alpha}$ tidy for $\left.\alpha\right|_{M_{\alpha}}$. To this end, let $W \in \mathcal{T}\left(\left.\alpha\right|_{M_{\alpha}}\right)$. Then there exists a compact open subgroup $O \subseteq G$ such that $O \cap M_{\alpha} \subseteq W$. Hence $\mathcal{K}_{O}=\left(O \cap M_{\alpha}\right)_{--} \subseteq$ $W_{--}$and thus $K \subseteq K_{O} \subseteq W_{--}$, using the fact that $W_{--}$is closed. The assertion follows. Since $M_{\alpha}=M_{\left.\alpha\right|_{M_{\alpha}}}$, we have $W_{-}=W=W_{+}$for each $W \in \mathcal{T}\left(\left.\alpha\right|_{M_{\alpha}}\right)$ by [1, Lemma 3.19], whence $\alpha(W)=W$ and thus $W_{--}=W$. Summing up, we have $K \subseteq \bigcap \mathcal{T}\left(\left.\alpha\right|_{M_{\alpha}}\right)$. But $\bigcap \mathcal{T}\left(\left.\alpha\right|_{M_{\alpha}}\right)=\{1\}$, as $\left.\alpha\right|_{M_{\alpha}}$ is tidy. Thus $K=\{1\}$. Using [1, Corollary 3.27] and $\overline{U_{\alpha}} \cap \overline{U_{\alpha^{-1}}} \subseteq P_{\alpha} \cap P_{\alpha^{-1}}=M_{\alpha}$, we also get $\{1\}=\bigcap \mathcal{T}\left(\left.\alpha\right|_{M_{\alpha}}\right)=\overline{U_{\left.\alpha\right|_{M_{\alpha}}}} \cap \overline{U_{\alpha^{-1} \mid M_{\alpha}}}=$ $\overline{U_{\alpha}} \cap \overline{U_{\alpha^{-1}}}=U_{0}$. 
Stimulated by the results in [1] (notably Propositions 3.7 and 3.21 and Theorem 3.32) and [9, Theorem 3.5(iii)], we now formulate various useful conclusions.

THEOREM. Let $G$ be a totally disconnected, locally compact group. Then $\alpha \in \operatorname{Aut}(G)$ is tidy if and only if $\left.\alpha\right|_{M_{\alpha}}$ is tidy. In this case, we have the following properties.

(a) $U_{0}=\{1\}$

(b) $U_{\alpha}$ and $U_{\alpha^{-1}}$ are closed.

(c) $U_{\alpha} \cap M_{\alpha}=U_{\alpha^{-1}} \cap M_{\alpha}=\{1\}$.

(d) Every compact open subgroup of $G$ satisfying $\mathbf{T} \mathbf{1}$ is tidy.

(e) $P_{\alpha}=U_{\alpha} \times M_{\alpha}$ and $P_{\alpha^{-1}}=U_{\alpha} \times M_{\alpha^{-1}}$ as topological groups.

(f) $U_{\alpha} M_{\alpha} U_{\alpha^{-1}}$ is an $\alpha$-stable, open subset of $G$ that contains every subgroup tidy for $\alpha$. The product map $\mu: U_{\alpha} \times M_{\alpha} \times U_{\alpha^{-1}} \rightarrow U_{\alpha} M_{\alpha} U_{\alpha^{-1}},(x, y, z) \mapsto x y z$ is a homeomorphism.

(g) $s_{G}\left(\alpha^{-1}\right)=s_{P_{\alpha}}\left(\left.\alpha^{-1}\right|_{P_{\alpha}}\right)=s_{U_{\alpha}}\left(\left.\alpha^{-1}\right|_{U_{\alpha}}\right)=s_{V_{--}}\left(\left.\alpha^{-1}\right|_{V_{--}}\right)$, for each subgroup $V \subseteq G$ tidy for $\alpha$, where each of $P_{\alpha}, V_{--}$and $U_{\alpha}$ are $\alpha$-invariant closed subgroups of $P_{\alpha}$.

(h) $s_{H}\left(\left.\alpha^{-1}\right|_{H}\right)=\Delta_{H}\left(\left.\alpha^{-1}\right|_{H}\right)$ for each $\alpha$-invariant closed subgroup $H$ of $P_{\alpha}$, where $\Delta_{H}$ : $\operatorname{Aut}(H) \rightarrow \mathbb{Q}^{+}$is the modular function. In particular, $s_{G}\left(\alpha^{-1}\right)=$ $\Delta_{U_{\alpha}}\left(\left.\alpha^{-1}\right|_{U_{\alpha}}\right)$.

(i) If $q: G \rightarrow Q$ is a quotient morphism with $\alpha(\operatorname{ker}(q))=\operatorname{ker}(q)$ and $\bar{\alpha}$ the induced automorphism of $Q$, then $q\left(U_{\alpha}\right)=U_{\bar{\alpha}}$.

Proof. We already know that tidiness of $\alpha$ entails tidiness of $\left.\alpha\right|_{M_{\alpha}}$. We assume that $\left.\alpha\right|_{M_{\alpha}}$ is tidy and show that this entails all of (a)-(i) as well as the tidiness of $\alpha$. In Lemma 2, we have already seen that (a) holds; (g), (h) and (i) are the analogues of [1, Proposition 3.7 and Proposition 3.21] in our setting, that remain valid by Lemma 1 above.

(b) Using (a) and [1, Corollary 3.30], we obtain $\overline{U_{\alpha}}=U_{0} U_{\alpha}=\{1\} U_{\alpha}=U_{\alpha}$. Replacing $\alpha$ with $\alpha^{-1}$ (whose restriction to $M_{\alpha}=M_{\alpha^{-1}}$ is tidy since so is $\alpha$ ), we see that also $U_{\alpha^{-1}}$ is closed.

(c) By (a), (b) and [1, Lemma 3.29], we have $U_{\alpha} \cap M_{\alpha}=\overline{U_{\alpha}} \cap M_{\alpha}=U_{0}=\{1\}$. Replacing $\alpha$ with $\alpha^{-1}$, we also obtain the second formula.

(d) Let $V \subseteq G$ be a compact open subgroup satisfying T1. We apply the tidying procedure from $[12, \S 2]$ to create a subgroup tidy for $\alpha$. Since $V$ satisfies T1, Step 1 of the algorithm outputs $V$. In Step 2a, one defines $K$ as above; thus $K=\{1\}$. In Step 3a, one defines

$$
V^{\prime \prime}:=\left\{v \in V: x v x^{-1} \in V K \text { for all } x \in K\right\}
$$

then $V^{\prime \prime}=V$ as $K=\{1\}$. Now $V^{\prime \prime} K=V$ is tidy by [12, Proposition 2.1].

$\alpha$ is tidy: Since every compact open subgroup of $G$ contains a compact open subgroup satisfying T1, by the first step of the algorithm just cited, and any such is tidy by (d), we see that $G$ has small subgroups tidy for $\alpha$.

(e) This can be proved as in [1, Theorem $3.32(6)$ and $\left.\left(6^{\prime}\right)\right]$.

(f) To see that $\mu$ is injective, suppose that $\mu(x, y, z)=\mu\left(x^{\prime}, y^{\prime}, z^{\prime}\right)$. Then $y^{-1} x^{-1} x^{\prime} y^{\prime}=z\left(z^{\prime}\right)^{-1} \in M_{\alpha} U_{\alpha} \cap U_{\alpha^{-1}}=P_{\alpha} \cap U_{\alpha^{-1}}=U_{0}=\{1\}$ by [1, Lemma 3.29], whence $z=z^{\prime}$ and $x y=x^{\prime} y^{\prime}$. Thus $x^{-1} x^{\prime}=y\left(y^{\prime}\right)^{-1} \in U_{\alpha} \cap M_{\alpha}=\{1\}$, using (c), and hence $x=x^{\prime}$ and $y=y^{\prime}$. Thus $\mu$ is injective. Let $V \subseteq G$ be tidy for $\alpha$. Then $V=V_{-} V_{+}$ where $V_{-}=\left(U_{\alpha} \cap V\right) V_{0}$ as a consequence of [1, Proposition 3.16] and likewise $V_{+}=$ $V_{0}\left(U_{\alpha^{-1}} \cap V\right)$. Here $U_{\alpha} \cap V, U_{\alpha^{-1}} \cap V$ and $V_{0}=M_{\alpha} \cap V$ are compact open subgroups of $U_{\alpha}, U_{\alpha^{-1}}$ and $M_{\alpha}$, respectively. Since $V=\left(U_{\alpha} \cap V\right) V_{0}\left(U_{\alpha^{-1}} \cap V\right)$, the map $\mu$ induces 
a continuous bijection between $W:=\left(U_{\alpha} \cap V\right) \times\left(M_{\alpha} \cap V\right) \times\left(U_{\alpha^{-1}} \cap V\right)$ and $V$. Both $W$ and $V$ being compact, we deduce that $\left.\mu\right|_{W} ^{V}$ is a homeomorphism. To verify that $\operatorname{im}(\mu)$ is open and $\mu$ a homeomorphism, let $\left(x_{0}, y_{0}, z_{0}\right) \in U_{\alpha} \times M_{\alpha} \times U_{\alpha^{-1}}$. As $M_{\alpha}$ normalizes $U_{\alpha}$, the formula $h(x, y, z):=\left(y_{0}^{-1} x y_{0}, y, z\right)$ defines a homeomorphism $h$ of $U_{\alpha} \times M_{\alpha} \times U_{\alpha^{-1}}$ onto itself. Left and right translations being homeomorphisms and $h^{-1}(W)$ being an identity neighbourhood, we now deduce from

$$
\left.x_{0} y_{0} \mu\right|_{W}(h(x, y, z)) z_{0}=\mu\left(x_{0} x, y_{0} y, z z_{0}\right), \quad \text { for all }(x, y, z) \in h^{-1}(W),
$$

that $\mu$ takes a neighbourhood of $\left(x_{0}, y_{0}, z_{0}\right)$ homeomorphically onto a neighbourhood of $\mu\left(x_{0}, y_{0}, z_{0}\right)$.

\section{REFERENCES}

1. U. Baumgartner and G. A. Willis, Contraction groups and scales of automorphisms of totally disconnected locally compact groups, Israel J. Math. 142 (2004), 221-248.

2. S. G. Dani and R. Shah, Contraction subgroups and semistable measures on $p$-adic Lie groups, Math. Proc. Camb. Phil. Soc. 110 (1991), 299-306.

3. H. Glöckner, Scale functions on p-adic Lie groups, Manuscr. Math. 97 (1998), 205-215.

4. H. Glöckner, Scale functions on Lie groups over local fields of positive characteristic, in preparation. 413-421.

5. H. Glöckner and G. A. Willis, Uniscalar p-adic Lie groups, Forum Math. 13 (2001),

6. W. Hazod, Remarks on (semi-) stable probabilities, in Probability Measures on Groups VII, Lecture Notes in Mathematics No. 1064 (Springer-Verlag, 1984), 182-203.

7. W. Hazod and E. Siebert, Automorphisms on a Lie group contracting modulo a compact subgroup and applications to semistable convolution semigroups, J. Theor. Probab. 1 (1988), 211-225. 73-90.

8. E. Siebert, Contractive automorphisms on locally compact groups, Math. Z. 191 (1986), 403-412.

9. J. S. P. Wang, The Mautner phenomenon for p-adic Lie groups, Math. Z. 185 (1984),

10. G. A. Willis, The structure of totally disconnected, locally compact groups, Math. Ann. 300 (1994), 341-363.

11. G. A. Willis, Further properties of the scale function on a totally disconnected group, J. Algebra 237 (2001), 142-164.

12. G. A. Willis, Tidy subgroups for commuting automorphisms of totally disconnected locally compact groups: An analogue of simultaneous triangularisation of matrices, New York J. Math. 10 (2004), 1-35. 